\title{
FORMULATION OF NANOPARTICLES OF ANTI-MIGRAINE DRUGS TRIPTANS BY COACERVATION METHOD
}

\author{
FARSHID A*, LAKSHMI CSR
}

Department of Pharmaceutics, Nargund College of Pharmacy, Bengaluru, Karnataka, India. Email: farshid_khezri@yahoo.com

Received: 29 May 2017, Revised and Accepted: 17 July 2017

ABSTRACT

Objective: Triptans are effective medicines used to treat migraine and certain other headaches. This study has been conducted to prepared nanoparticles (NPs) of triptans by coacervation technique using a different drug to polymer ratios and evaluated.

Methods: Polymers such as albumin and gelatin and cross-linking agent were used at different levels to prepare triptan NPs with high entrapment efficiency (EE) and low particle size (PS). Completion of the reaction was confirmed by infrared spectra. NPs were evaluated for shape and surface morphology, polydispersity index, zeta potential, EE, and PS distribution.

Results: Among all the formulation, $\mathrm{A}_{\mathrm{A} 5}$ formulation of triptan-loaded albumin NPs (ANPs) has small PS and high EE.

Conclusion: In this study, we have found that the ANPs in the presence of $4 \%$ glutaraldehyde as a cross-linking agent could be used as a delivery vehicle of triptans.

Keywords: Triptan, Nanoparticles, Albumin and Gelatin, Coacervation method.

(C) 2017 The Authors. Published by Innovare Academic Sciences Pvt Ltd. This is an open access article under the CC BY license (http://creativecommons. org/licenses/by/4. 0/) DOI: http://dx.doi.org/10.22159/ajpcr.2017.v10i11.20302

\section{INTRODUCTION}

Migraine a headache is a neurological disorder often initiated by a trigger and characterized by a headache, which may be accompanied by a variety of multiple organ/system symptoms such as nausea, allodynia, vomiting, and urinary frequency. They are selective agonists of specific 5-hydroxytryptamine (5-HT) receptors such as the 5-HT1B and 5-HT1D receptors. Migraine is a severe, painful headache that is often preceded or accompanied by sensory warning signs such as flashes of light, blind spots, tingling in the arms and legs, nausea, vomiting, and increased sensitivity to light and sound. The pain that migraines bring can last for hours or even days [1,2].

Triptans, also called as serotonin 5-HT receptor agonists, are used to treat migraine and certain other headaches. They may be taken subcutaneously, orally as tablets, capsules, or quick-dissolving wafers, or intranasally as a spray [3].

The use of polymeric nanoparticles (NPs) in drug delivery generally increases the stability of the pharmaceutical agents, and it can be easily and cheaply fabricated in large quantities by a multitude of methods [4].

NPs have been extensively investigated in biomedical and biotechnological areas and, especially, in drug delivery systems for drug targeting. The advantages of targeted drug delivery to the specific site of the body paved the way for applying NPs to achieve this type of drug delivery. Much attention has been provided to nonparenteral routes such as oral, pulmonary, nasal, and ophthalmic delivery of the drugs $[5,6]$.

The present research work deals with the design of targeted NPs of drugs such as almotriptan, naratriptan, frovatriptan, and zolmitriptan, (second-generation triptans) using coacervation technique, using albumin and gelatin (biodegradable polymers). The NPs so formed have high entrapment efficiency (EE) and low particle size (PS).

\section{METHODS}

Almotriptan, zolmitriptan, naratriptan, and frovatriptan were received as a gift samples from Apotex Research Private Limited, Bangalore. Albumin and gelatin were procured from DMV, Fonterra excipients, India, 4\% glutaraldehyde, hydrochloric acid and Sodium hydroxide were purchased from S.D. Fine Chem. Ltd.

\section{Analytical method}

About $100 \mathrm{mg}$ of Almotriptan was dissolved in $\mathrm{pH} 5.5$ phosphate buffer in a $100 \mathrm{ml}$ volumetric flask and made up to $100 \mathrm{ml}$ with the same. Aliquots from this stock solution were taken, and dilutions were done using distilled water to obtain concentrations of $2,4,6,8$, and $10 \mu \mathrm{g} / \mathrm{ml}$. Absorbances of these solutions were measured at 227 ( $\lambda_{\max }$ of almotriptan). The data are presented in Tables 1-4. Graphical representation is given in Figs 1-4.

The same procedure has been repeated with the zolmitriptan, naratriptan, and frovatriptan at suitable $\lambda_{\text {max }}(222,223$, and $244 \mathrm{~nm}$, respectively).

\section{Drug-excipient compatibility study}

Drug-excipient interaction plays a key role in the release of drug from the formulation. Fourier transform infrared spectroscopy (FTIR) has been used to study the physical and chemical interactions between

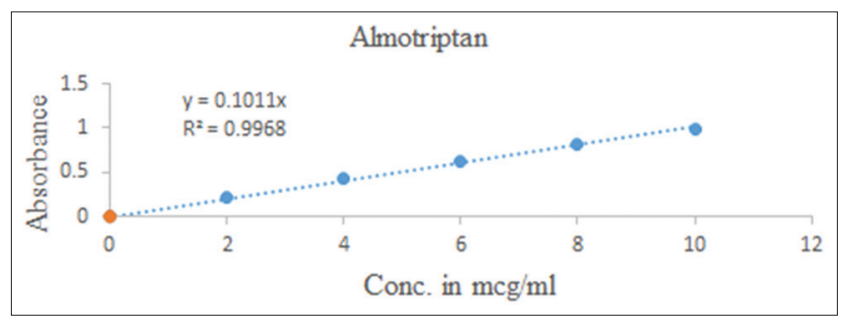

Fig. 1: Calibration curve of almotriptan 


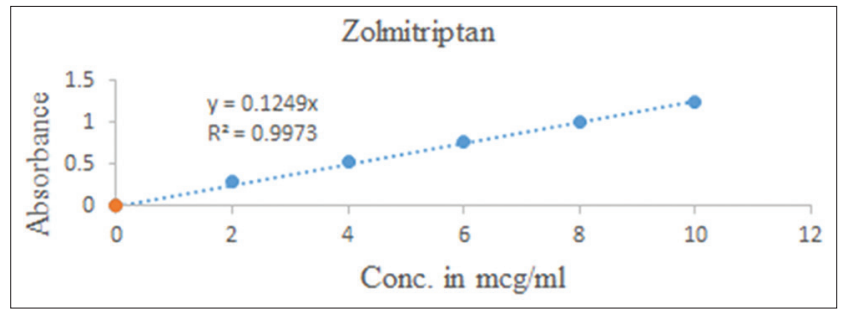

Fig. 2: Calibration curve of zolmitriptan

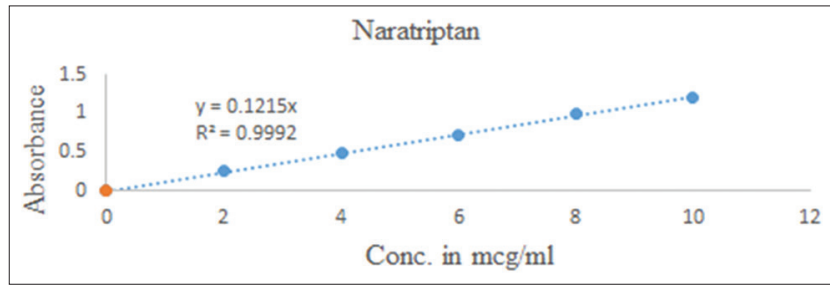

Fig. 3: Calibration curve of naratriptan

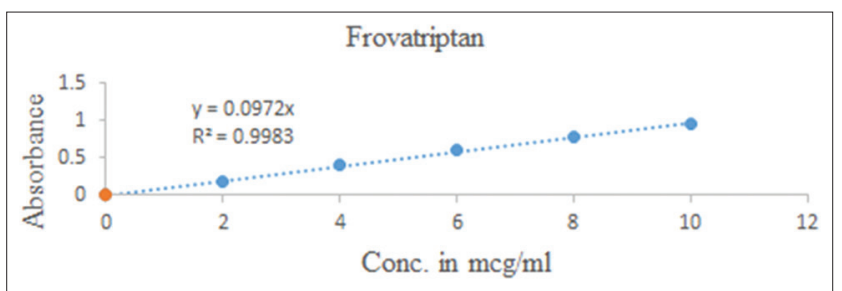

Fig. 4: Calibration curve of frovatriptan

the drug and the excipients used. FTIR spectra of albumin, gelatin and physical mixture of triptans with albumin and gelatin were recorded using $\mathrm{KBr}$ pellet method on FTIR instrument.

\section{Preparation of triptan-loaded albumin NPs (ANPs)}

ANPs containing the drugs (almotriptan) were prepared by the coacervation technique $[7,8]$. The accurately weighed amount of almotriptan $5 \mathrm{mg}$ and egg albumin at various ratios $(1: 1,1: 2$, and 1:3) were dissolved in $50 \mathrm{ml}$ of distilled water. Then, the solution was stirred using magnetic stirrer and required an amount of acetone was added dropwise from a syringe until the solution becomes turbid. The egg albumin was cross linked by adding $4 \%$ glutaraldehyde. Then, the $\mathrm{pH}$ was adjusted to 5.5 with the help of required amount of $0.1 \mathrm{~N}$ hydrochloric acid.

The solution was mixed continuously for $3 \mathrm{hrs}$ at $4000 \mathrm{rpm}$ at room temperature using mechanical stirrer. After cross-linking, the egg ANPs were subjected to sonication for 5 minutes. Then, the nanoparticles were centrifuged at $5000 \mathrm{rpm}$ for 30 minutes. After centrifugation, the supernatant liquid was removed, and the suspension was washed 3-4 times with acetone and then finally dried in a vacuum dryer (Table 5).

The best procedure has been repeated with the zolmitriptan, naratriptan, and frovatriptan.

Preparation of Triptan-loaded gelatin NPs (G-NPs) by two-step modified desolvation method

G-NPs containing the drugs almotriptan were prepared by the coacervation technique [9-12]. The accurately weighed amount of almotriptan and gelatin type $A$ at various ratios (1:1, 1:2, and 1:3) were dissolved in distilled water $(50 \mathrm{ml})$ under constant heating temperature range. Acetone was added to the gelatin solution as a desolvating agent to precipitate the high molecular weight (HMW) gelatin. The supernatant was discarded and the HMW gelatin re-dissolved by
Table 1: Calibration curve data for almotriptan in phosphate buffer pH 5.5 at $\lambda_{\text {max }}$ of $227 \mathrm{~nm}$

\begin{tabular}{ll}
\hline Concentration & Absorbance \\
\hline 0 & $0 \pm 0.01$ \\
2 & $0.209 \pm 0.03$ \\
4 & $0.430 \pm 0.04$ \\
6 & $0.622 \pm 0.02$ \\
8 & $0.823 \pm 0.01$ \\
10 & $0.978 \pm 0.05$ \\
\hline${ }^{*}=3$. Values are Mean+SD. SD: Standard deviation
\end{tabular}

${ }^{*} \mathrm{n}=3$. Values are Mean \pm SD. SD: Standard deviation

Table 2: Calibration curve data for zolmitriptan in phosphate buffer pH 5.5 at $\lambda_{\text {max }}$ of $222 \mathrm{~nm}$

\begin{tabular}{ll}
\hline Concentration & Absorbance \\
\hline 0 & $0 \pm 0.02$ \\
2 & $0.280 \pm 0.03$ \\
4 & $0.536 \pm 0.01$ \\
6 & $0.759 \pm 0.04$ \\
8 & $0.991 \pm 0.02$ \\
10 & $1.230 \pm 0.03$ \\
\hline$*_{n}=3$. Values are Mean \pm SD. SD: Standard deviation
\end{tabular}

Table 3: Calibration curve data for naratriptan in phosphate buffer pH 5.5 at $\lambda_{\max }$ of $223 \mathrm{~nm}$

\begin{tabular}{ll}
\hline Concentration & Absorbance \\
\hline 0 & $0 \pm 0.01$ \\
2 & $0.255 \pm 0.01$ \\
4 & $0.488 \pm 0.03$ \\
6 & $0.719 \pm 0.02$ \\
8 & $0.993 \pm 0.01$ \\
10 & $1.202 \pm 0.04$ \\
\hline$*_{\mathrm{n}=3 .}$ Values are Mean+SD. SD: Standard deviation
\end{tabular}

Table 4: Calibration curve data for frovatriptan in phosphate buffer $\mathrm{pH} 5.5$ at $\lambda_{\max }$ of $244 \mathrm{~nm}$

\begin{tabular}{ll}
\hline Concentration & Absorbance \\
\hline 0 & $0 \pm 0.01$ \\
2 & $0.189 \pm 0.01$ \\
4 & $0.407 \pm 0.02$ \\
6 & $0.594 \pm 0.02$ \\
8 & $0.790 \pm 0.04$ \\
10 & $0.950 \pm 0.02$ \\
\hline$*_{n}=3$. Values are Mean ${ }^{\text {SD. SD: Standard deviation }}$
\end{tabular}

addition $50 \mathrm{~mL}$ distilled water and stirring at $600 \mathrm{rpm}$ under constant heating. Acetone was added dropwise to form NPs. 4\% glutaraldehyde solution was used as a cross-linking agent. At the end of the process, the $\mathrm{pH}$ of the gelatin solution was adjusted to 5.5 with $1 \mathrm{~N} \mathrm{HCl}$ and stirred for $12 \mathrm{hrs}$ at $600 \mathrm{rpm}$. The particles were purified by three-fold centrifugation and dispersed in acetone (30\%) with Milli-Q water. After the last redispersion, the acetone was evaporated using concentrator (speed vacuum). Finally, after stirring for $12 \mathrm{hrs}$, the particles were purified by three-fold centrifugation (16000 rpm for 20 minutes) and dispersed in acetone/water (30/70). The purified NPs are stored as a dispersion in highly purified water (conductivity $<0.04 \mu \mathrm{s} / \mathrm{cm}$ ) at $4-8^{\circ} \mathrm{C}$. The following parameters were changed to study their effect on the characteristics of the NPs: Concentration of gelatin (Type A) and crosslinking agent ( $4 \%$ glutaraldehyde) (Table 6).

The best procedure has been repeated with the zolmitriptan, naratriptan, and frovatriptan. 
Characterization of NPs

\section{Shape and surface morphology}

The shape and surface morphology of the albumin and G-NPs were visualized by scanning electron microscopy (SEM) (LEO-430 Cambridge and U.K). The samples were prepared by lightly sprinkling NPs on double-sided adhesive tape on an aluminum stub. The stubs were then coated with gold to a thickness of 200-500 Å under an argon atmosphere using a gold sputter module in a high vacuum evaporator [13]. The samples were then randomly scanned, and photomicrographs were taken at different magnifications with SEM.

\section{PS and zeta potential measurement}

PS was measured with the help of HORIBA Scientific Nano Partica, NP analyzer SZ-100. For the determination of PS, samples were prepared by 10 -fold dilution of $1 \mathrm{ml}$ of the nanoparticulate suspension with distilled water. The analysis was performed in triplicate [14]

The average PS, polydispersity index and Zeta potential were measured by HORIBA scientific Nano Partica, NPs analyzer SZ-100.

\section{Drug EE}

The EE of the formulation was determined on the centrifugation of a fixed quantity of the aqueous nanoparticulate suspension (about $2 \mathrm{ml}$ ) at $12000 \mathrm{rpm}$ for 30 minutes at $20^{\circ} \mathrm{C}$ (SIGMA 3-18K, Sartorius). The absorbance of the unencapsulated drug in the supernatant was evaluated using UV-VIS spectrophotometer (UV-1800 Pharma Spec, Shimadzu).

The drug entrapment of the NPs was calculated by the following equation [14].
Intialamount of thedrugadded -

$\%$ Drug entrapment efficiency $=\frac{\text { Amount of druginsupernatant }}{\text { Intialamount of thedrugadded }} \times 100$

\section{RESULTS AND DISCUSSION}

Analytical method

The calibration curve for Almotriptan, Zolmitriptan, Naratriptan and Frovatriptan in $\mathrm{pH} 5.5$ buffer was linear in concentration range between $2-10 \mu \mathrm{g} / \mathrm{ml}$ at $227,222,223,244 \mathrm{~nm}$ respectively. The data is presented in (Tables 1-4) and graphical representation is given in (Fig. 1-4) respectively

\section{Drug-excipients compatibility studies by FTIR}

FTIR techniques have been used here to study the physical and chemical interaction between drug and excipients used. In the present study, it has been observed that there is no chemical interaction between Almotriptan, Zolmitriptan, Naratriptan, Frovatriptan and the excipients used, thus indicating compatibility of the drugs and excipients (Figs. 5-8) IR spectrum of Albumin and Gelatin polymer were present in (Fig. 9).

$P S$, zeta potential measurement of NPS

The values for the average PS, zeta potential and polydispersity index are tabulated in Tables 7-10.

The drug and albumin polymer, ratio of $1: 2$ with $200 \mu \mathrm{l}$ of $4 \%$ glutaraldehyde as cross linker $\left(\mathrm{A}_{\mathrm{A}}\right)$ showed $\mathrm{PS}$ of $48.8 \mathrm{~nm}$, polydispersity index of 0.51 , and zeta potential of $+29.0 \mathrm{mV}$. Results were shown in Fig.10.

Table 5: Experimental control factors and different levels of polymer (albumin) and $4 \%$ glutaraldehyde for almotriptan loaded-albumin nanoparticles $\left(A_{A 1}-A_{A 9}\right)$ formulation

\begin{tabular}{|c|c|c|c|c|c|c|c|c|c|c|}
\hline SNo. & Ingredients & $\mathbf{A}_{\mathrm{A} 1}$ & $\mathrm{~A}_{\mathrm{A} 2}$ & $\mathrm{~A}_{\mathrm{A} 3}$ & $\mathbf{A}_{\mathrm{A} 4}$ & $\mathbf{A}_{\mathrm{A} 5}$ & $\mathrm{~A}_{\mathrm{A} 6}$ & $\mathbf{A}_{\mathrm{A} 7}$ & $\mathbf{A}_{\mathrm{A} 8}$ & $\mathbf{A}_{\mathrm{A} 9}$ \\
\hline 1 & Drug:polymer concentration & 1:1 & $1: 2$ & $1: 3$ & $1: 1$ & $1: 2$ & $1: 3$ & $1: 1$ & $1: 2$ & $1: 3$ \\
\hline 2 & Acetone & 2 & 2 & 2 & 2 & 2 & 2 & 2 & 2 & 2 \\
\hline 3 & $4 \%$ glutaraldehyde $(\mu \mathrm{l})$ & 150 & 150 & 150 & 200 & 200 & 200 & 250 & 250 & 250 \\
\hline 4 & Distilled water (ml) & 50 & 50 & 50 & 50 & 50 & 50 & 50 & 50 & 50 \\
\hline 5 & Stirring speed (rpm) & 1500 & 1500 & 1500 & 1500 & 1500 & 1500 & 1500 & 1500 & 1500 \\
\hline
\end{tabular}

$0.1 \mathrm{~N} \mathrm{HCl}$ is added till the $\mathrm{pH}$ of the solution reaches 5.5

Table 6: Experimental control factors and different levels of polymer (gelatin) and 4\% glutaraldehyde for almotriptan loaded gelatin nanoparticles $\left(A_{G 1}-A_{G 9}\right)$ formulation

\begin{tabular}{|c|c|c|c|c|c|c|c|c|c|c|}
\hline S.No. & Ingredients & $A_{G 1}$ & $\mathbf{A}_{\mathrm{G} 2}$ & $\mathbf{A}_{\mathrm{G} 3}$ & $\mathbf{A}_{\mathrm{G} 4}$ & $\mathbf{A}_{\mathrm{G} 5}$ & $\mathbf{A}_{\mathrm{G} 6}$ & $\mathbf{A}_{\mathrm{G} 7}$ & $\mathbf{A}_{\mathrm{G} 8}$ & $\mathbf{A}_{\mathrm{G} 9}$ \\
\hline 1 & Drug:polymer concentration & $1: 1$ & $1: 2$ & $1: 3$ & $1: 1$ & $1: 2$ & $1: 3$ & $1: 1$ & $1: 2$ & $1: 3$ \\
\hline 2 & $4 \%$ glutaraldehyde $(\mu \mathrm{l})$ & 150 & 150 & 150 & 200 & 200 & 200 & 250 & 250 & 250 \\
\hline 3 & Distilled water (ml) & 50 & 50 & 50 & 50 & 50 & 50 & 50 & 50 & 50 \\
\hline 4 & Acetone $(\mathrm{ml})$ & 75 & 75 & 75 & 75 & 75 & 75 & 75 & 75 & 75 \\
\hline 5 & Stirring speed (rpm) & 1500 & 1500 & 1500 & 1500 & 1500 & 1500 & 1500 & 1500 & 1500 \\
\hline 6 & Temperature & 50 & 50 & 50 & 50 & 50 & 50 & 50 & 50 & 50 \\
\hline
\end{tabular}

$0.1 \mathrm{~N} \mathrm{HCl}$ is added till the $\mathrm{pH}$ of the solution reaches 5.5

Table 7: Data for particle size, entrapment efficiency, zeta potential, polydispersity index for the formulation of almotriptan, at variable levels of albumin, and $4 \%$ glutaraldehyde at low concentration: $A_{A 1}, A_{A 2}, A_{A 3}$, medium concentration: $A_{A 4}, A_{A 5}, A_{A 6}$, and high concentration: $A_{A 7}, A_{A 8}, A_{A}$, respectively

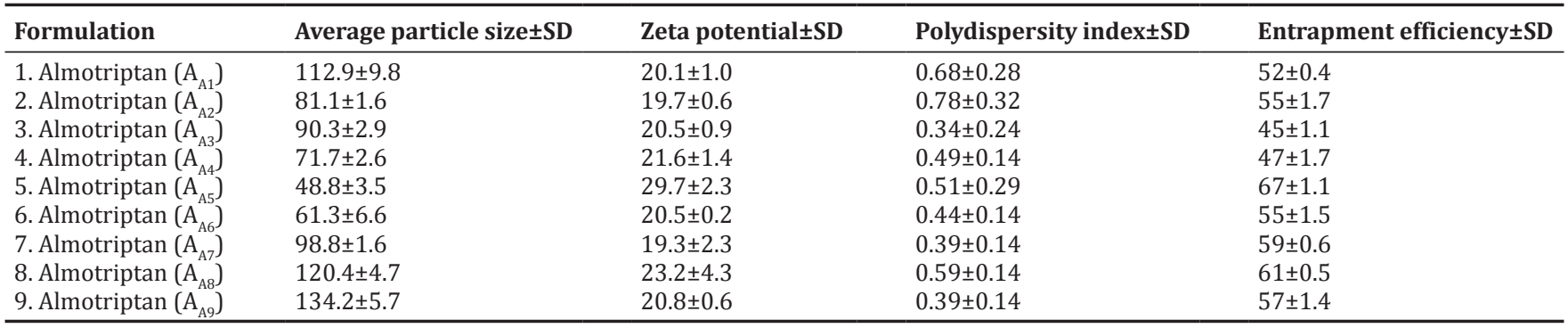

$\mathrm{n}=3$. Values are mean \pm SD. SD: Standard deviation 


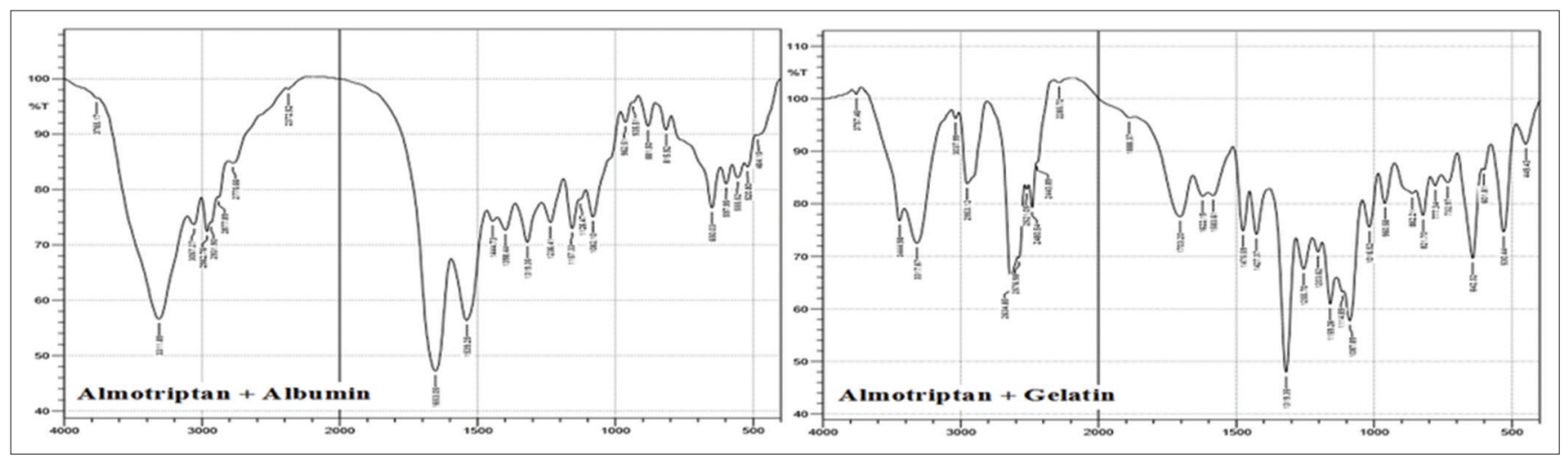

Fig. 5: Fourier transform infrared spectra of almotriptan + albumin and almotriptan + gelatin

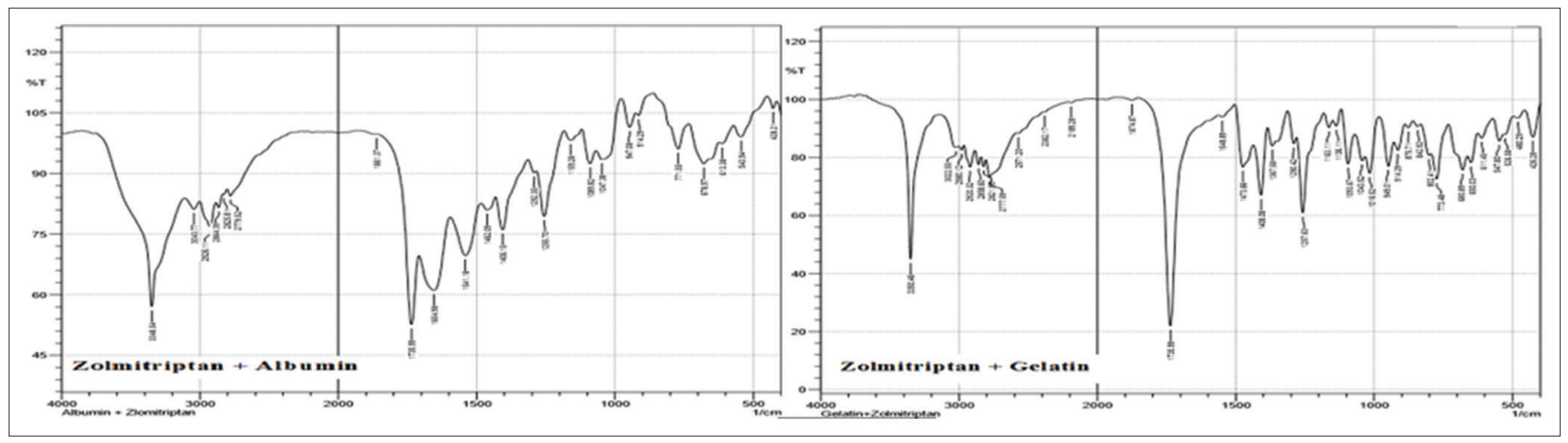

Fig. 6: Fourier transform infrared spectra of zolmitriptan + albumin and zolmitriptan + gelatin

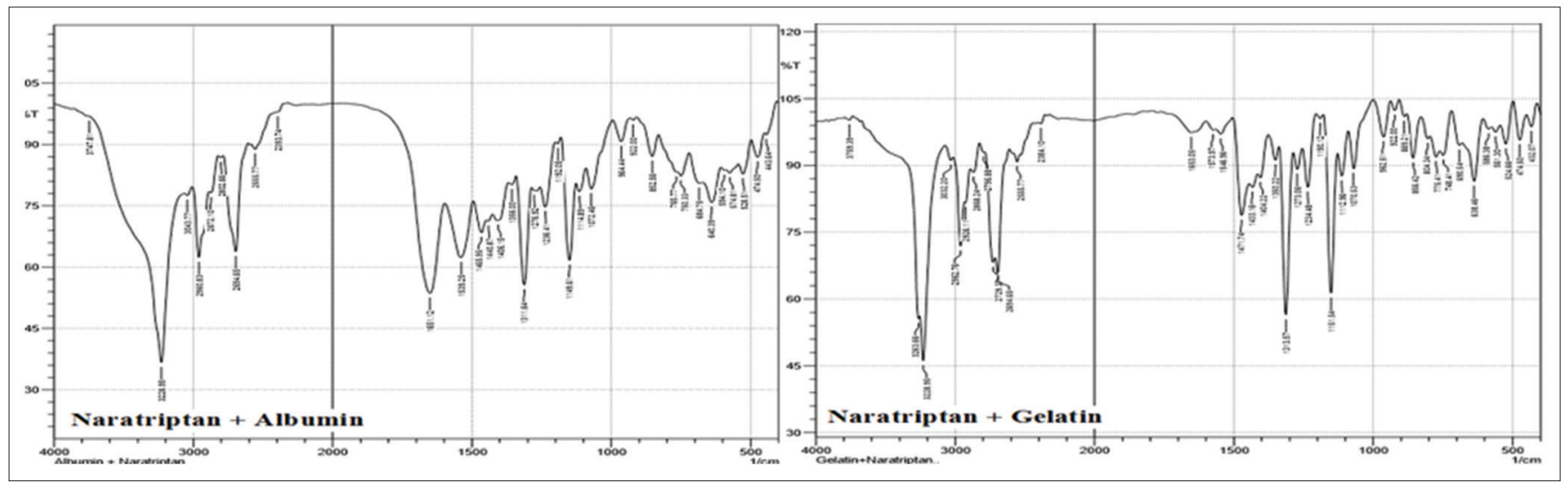

Fig. 7: Fourier transform infrared spectra of naratriptan + albumin and naratriptan + gelatin

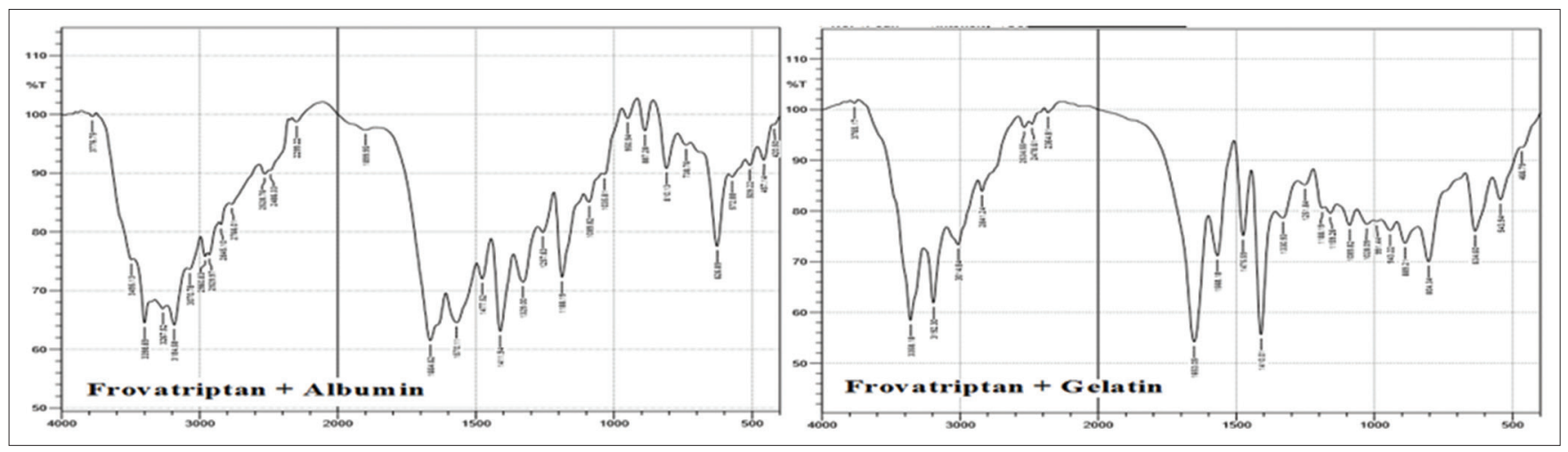

Fig. 8: Fourier transform infrared spectra of frovatriptan + albumin and frovatriptan + gelatin 


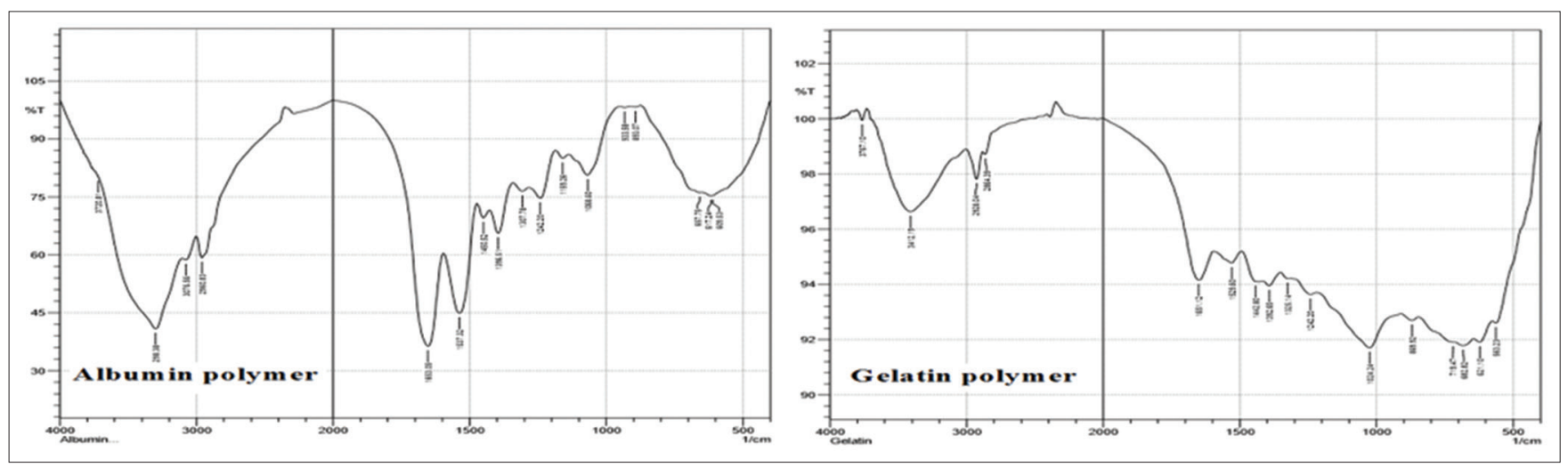

Fig. 9: Infrared spectra of albumin and gelatin polymer

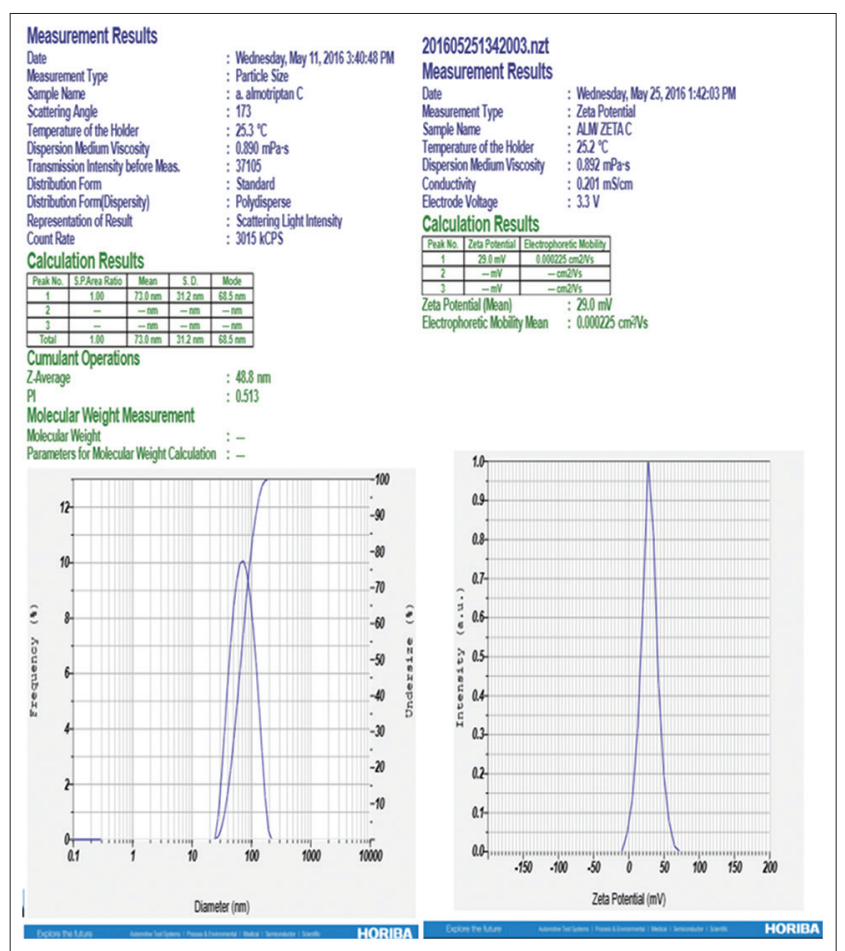

Fig. 10: Average particle size and zeta potential of almotriptan loaded albumin nanoparticles

The drug and gelatin polymer, ratio of $1: 2$ with $200 \mu \mathrm{l}$ of $4 \%$ glutaraldehyde as a cross linker $\left(A_{G 5}\right)$ showed PS of $96.7 \mathrm{~nm}$, polydispersity index of 0.59 , and zeta potential of $+23.4 \mathrm{mV}$. Results were shown in Fig. 11.

From the above formulations the drug and polymer ratio of $1: 2$ and $200 \mu \mathrm{l}$ of $4 \%$ glutaraldehyde showed the best results for both albumin and G-NPs formulations of all the drugs.

Therefore, we decided to repeat the best procedure for the formulation of albumin and G-NPs $\left(A_{A 5}\right.$ and $\left.A_{G 5}\right)$ of zolmitriptan, naratriptan, and frovatriptan. The results of which are shown in Tables 8 and 10, respectively.

In general, particle aggregation is less likely to occur in cases of high zeta potential due to electric repulsion.

As we are finding Formulation No. 8, it is given as the best result, so we are following the same formulation, (Table 11) for zolmitriptan $\left(\mathrm{Z}_{\mathrm{A}}\right)$ and naratriptan $\left(\mathrm{N}_{\mathrm{A}}\right)$ and frovatriptan $\left(\mathrm{F}_{\mathrm{A}}\right)$.

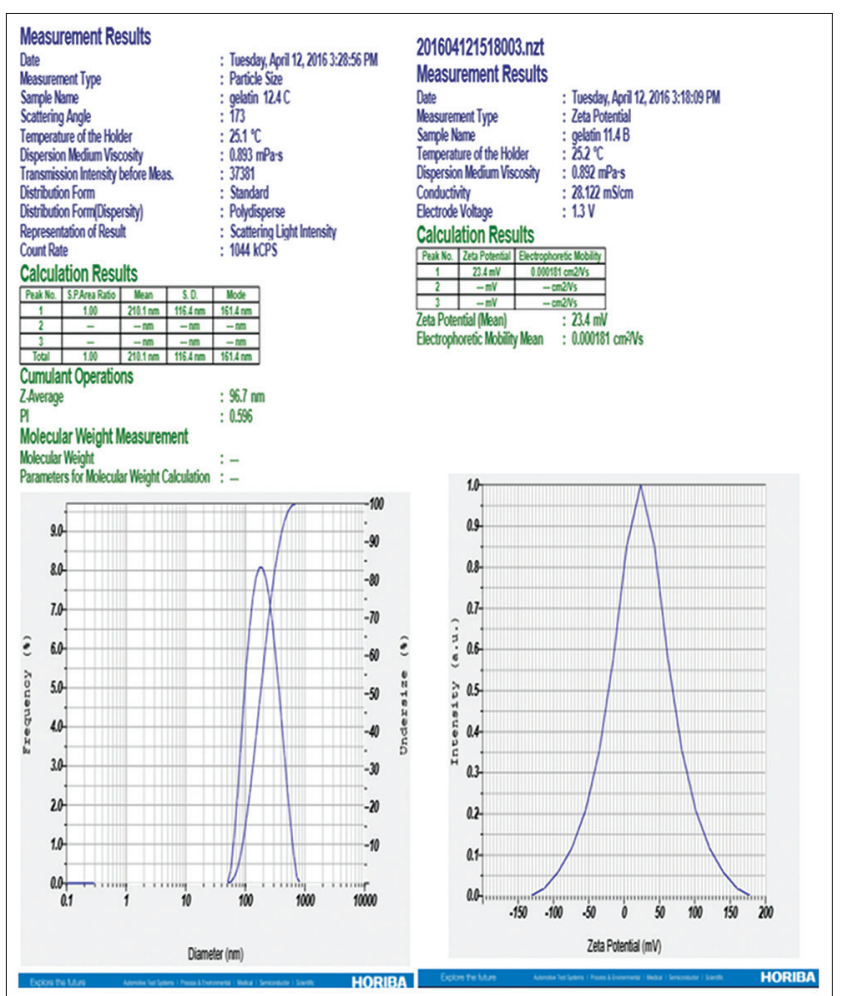

Fig. 11: Average particle size and zeta potential of almotriptan loaded gelatin nanoparticles

As we are finding Formulation No. $8\left(\mathrm{~A}_{\mathrm{G} 5}\right)$, it is given as the best result, so we are following the same formulation, (Table 12) for zolmitriptan $\left(\mathrm{Z}_{\mathrm{G}}\right)$ and naratriptan $\left(\mathrm{N}_{\mathrm{G}}\right)$ and frovatriptan $\left(\mathrm{F}_{\mathrm{G}}\right)$.

EE

EE of the triptan-loaded albumin and G-NPs was analyzed and the data were shown in Tables 7-10. $\mathrm{A}_{\mathrm{G} 5}$ showed an average drug EE of $75.4 \pm 0.8 \%$, which is highest among all the formulations.

\section{SEM}

The shape and surface morphology of the almotriptan-loaded G-NPs and almotriptan-loaded ANPs were visualized by SEM.

The SEM image of Gelatin polymer, pure drug (Almotriptan) and Almotriptan-loaded Gelatin nanoparticles are present in (Figs. 12 and 13) respectively. 
Table 8: Data for particle size, entrapment efficiency, zeta potential, polydispersity index for the formulation of zolmitriptan, naratriptan, frovatriptan based on best formulation

\begin{tabular}{lllll}
\hline Formulation & Average particle size \pm SD & Zeta potential \pm SD & Polydispersity index \pm SD & Entrapment efficiency \pm SD \\
\hline 1. Zolmitriptan $\left(\mathrm{Z}_{\mathrm{A}}\right)$ & $79.1 \pm 4.5$ & $21.1 \pm 0.6$ & $0.46 \pm 0.23$ & $68 \pm 1.1$ \\
2. Naratriptan $\left(\mathrm{N}_{\mathrm{A}}\right)$ & $69.5 \pm 2.4$ & $22.2 \pm 0.7$ & $0.51 \pm 0.14$ & $65 \pm 1.7$ \\
3. Frovatriptan $\left(\mathrm{F}_{\mathrm{A}}\right)$ & $55.5 \pm 5.7$ & $24.1 \pm 0.1$ & $0.44 \pm 0.17$ & $59 \pm 0.4$ \\
\hline
\end{tabular}

$\mathrm{n}=3$. Values are mean \pm SD. SD: Standard deviation

Table 9: Data for particle size, entrapment efficiency, zeta potential, polydispersity index for the formulation of almotriptan, at variable levels of gelatin, and $4 \%$ glutaraldehyde at low concentration: $A_{G 1}, A_{G 2}, A_{G 3}$, medium concentration $A_{G 4}, A_{G 5}, A_{G 6}$, and high concentration: $A_{G 7}, A_{G 8}, A_{G 9}$, respectively

\begin{tabular}{lllll}
\hline Formulation & Average particle size \pm SD & Zeta potential \pm SD & Polydispersity index \pm SD & Entrapment efficiency \pm SD \\
\hline 1. Almotriptan $\left(A_{\mathrm{G} 1}\right)$ & $136.9 \pm 9.8$ & $20.1 \pm 1.0$ & $0.58 \pm 0.28$ & $52 \pm 0.4$ \\
2. Almotriptan $\left(\mathrm{A}_{\mathrm{G} 2}\right)$ & $151.1 \pm 1.6$ & $21.7 \pm 0.6$ & $0.38 \pm 0.32$ & $65 \pm 1.7$ \\
3. Almotriptan $\left(\mathrm{A}_{\mathrm{G} 3}\right)$ & $142.3 \pm 2.9$ & $19.5 \pm 0.9$ & $0.34 \pm 0.24$ & $55 \pm 1.1$ \\
4. Almotriptan $\left(\mathrm{A}_{\mathrm{G} 4}\right)$ & $103.7 \pm 2.6$ & $22.6 \pm 1.4$ & $0.39 \pm 0.14$ & $67 \pm 1.7$ \\
5. Almotriptan $\left(\mathrm{A}_{\mathrm{G} 5}\right)$ & $96.7 \pm 3.5$ & $23.4 \pm 2.3$ & $0.59 \pm 0.19$ & $75 \pm 0.4$ \\
6. Almotriptan $\left(\mathrm{A}_{\mathrm{G} 6}\right)$ & $106.6 \pm 2.3$ & $26.6 \pm 1.4$ & $0.56 \pm 1.14$ & $71 \pm 1.4$ \\
7. Almotriptan $\left(\mathrm{A}_{\mathrm{G} 7}\right)$ & $111.6 \pm 0.4$ & $22.6 \pm 1.4$ & $0.42 \pm 1.33$ & $68 \pm 1.4$ \\
8. Almotriptan $\left(\mathrm{A}_{\mathrm{G} 8}\right)$ & $124.6 \pm 1.9$ & $23.6 \pm 1.4$ & $0.51 \pm 0.34$ & $69 \pm 1.4$ \\
9. Almotriptan $\left(\mathrm{A}_{\mathrm{G} 9}\right)$ & $171.3 \pm 6.6$ & $20.5 \pm 0.2$ & $0.54 \pm 0.54$ & $75 \pm 1.5$ \\
\hline
\end{tabular}

$\mathrm{n}=3$. Values are mean \pm SD. SD: Standard deviation

Table 10: Data for particle size, entrapment efficiency, zeta potential, polydispersity index, formulation of zolmitriptan, naratriptan, frovatriptan based on best formulation

\begin{tabular}{llll}
\hline Formulation & Average particle size \pm SD & Zeta potential \pm SD & Polydispersity index \pm SD \\
\hline 1. Zolmitriptan $\left(\mathrm{Z}_{\mathrm{G}}\right)$ & $95.1 \pm 4.5$ & $21.1 \pm 0.6$ & $0.46 \pm 0.23$ \\
2. Naratriptan $\left(\mathrm{N}_{\mathrm{G}}\right)$ & $99.5 \pm 2.4$ & $22.2 \pm 0.7$ & $0.51 \pm 0.14$ \\
3. Frovatriptan $\left(\mathrm{F}_{\mathrm{G}}\right)$ & $85.5 \pm 5.7$ & $24.1 \pm 0.1$ & $0.44 \pm 0.17$ \\
\hline
\end{tabular}

$\mathrm{n}=3$. Values are mean \pm SD. SD: Standard deviation

Table 11: Data for formulation of zolmitriptan $\left(\mathrm{Z}_{\mathrm{A}}\right)$, naratriptan $\left(\mathrm{N}_{\mathrm{A}}\right)$, and frovatriptan $\left(\mathrm{F}_{\mathrm{A}}\right)$

\begin{tabular}{lll}
\hline S.No. & Ingredients & $\mathbf{Z}_{\mathbf{A}^{\prime}}, \mathbf{N}_{\mathbf{A}}, \mathbf{F}_{\mathbf{A}}$ \\
\hline 1 & Drug:polymer (albumin) concentration $(\mathrm{mg})$ & $1: 2$ \\
2 & 4\% Glutaraldehyde $(\mu \mathrm{l})$ & 200 \\
3 & Distilled water $(\mathrm{ml})$ & 50 \\
4 & Acetone $(\mathrm{ml})$ & 2 \\
5 & Stirring speed $(\mathrm{rpm})$ & 1500 \\
\hline
\end{tabular}

$0.1 \mathrm{~N} \mathrm{HCl}$ is added till the $\mathrm{pH}$ of the solution reaches 5.5

Table 12: Data for formulation of zolmitriptan $\left(\mathrm{Z}_{\mathrm{G}}\right)$, naratriptan $\left(\mathrm{N}_{\mathrm{G}}\right)$, and frovatriptan $\left(\mathrm{F}_{\mathrm{G}}\right)$

\begin{tabular}{lll}
\hline S.No. & Ingredients & $\mathbf{Z}_{\mathbf{G}}, \mathbf{N}_{\mathbf{G}}, \mathbf{F}_{\mathbf{G}}$ \\
\hline 1 & Drug: polymer (gelatin) concentration $(\mathrm{mg})$ & $1: 2$ \\
2 & 4\% glutaraldehyde ethanol $(\mu \mathrm{l})$ & 200 \\
3 & Distilled water $(\mathrm{ml})$ & 50 \\
4 & Acetone $(\mathrm{ml})$ & 75 \\
5 & Stirring speed $(\mathrm{rpm})$ & 1500 \\
6 & Temperature & 50 \\
\hline
\end{tabular}

$0.1 \mathrm{~N} \mathrm{HCl}$ is added till the $\mathrm{pH}$ of the solution reaches 5.5

The SEM image of Albumin polymer and Almotriptan-loaded Albumin nanoparticles (A-NP) are present in (Fig. 14).

It revealed that the NPs were spherical in shape and have a smooth surface.

\section{DISCUSSION}

Coacervation is a new method by which stable nanoparticles can be prepared with a Zeta potential of $+29.1 \mathrm{mV}$ which was confirmed
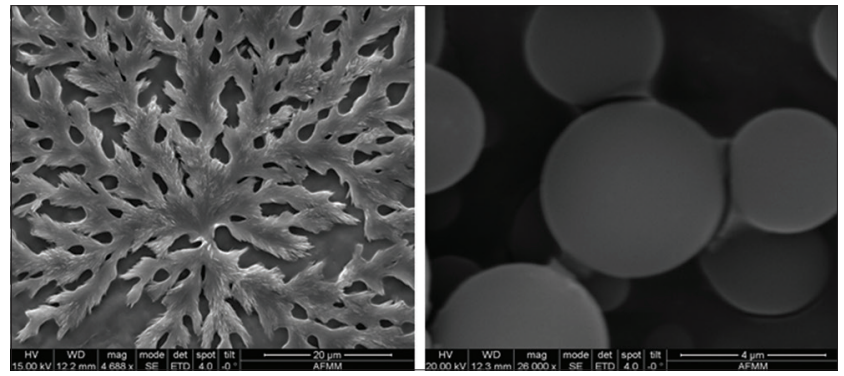

Fig. 12: Scanning electron microscopy images of gelatin and drug (almotriptan)

through HORIBA Scientific Nano Partica, NP analyzer SZ-100 (Table 8). Electrophoresis confirms the particles are positively charged in both albumin and G-NP.

Triptan-loaded albumin and G-NPs were successfully formulated by the coacervation method, and the effects of process parameters on NPs size, zeta potential, and polydispersity were characterized using the HORIBA scientific Nano Partica, NP analyzer SZ-100.

Preformulation studies such as standard graph and compatibility studies between polymer and drug were determined.

Results indicated that the of triptan-loaded ANPs, $A_{A 5}$ formulation has EE of $67.1 \pm 1.1 \%$, PS of $49 \mathrm{~nm}$, polydispersity index of 0.51 , and zeta potential of $+29.1 \mathrm{mV}$. Similarly, in triptan-loaded G-NPs $\mathrm{A}_{\mathrm{G} 5}$ was selected as the optimum formulation in which the EE is $75.4 \pm 0.8 \%$, PS is around $96 \mathrm{~nm}$, polydispersity index of 0.59 , and zeta potential of $+23.5 \mathrm{mV}$, which may be attributed to the positive charges on the both polymer matrices (albumin and gelatin) and surfactant mixture. 


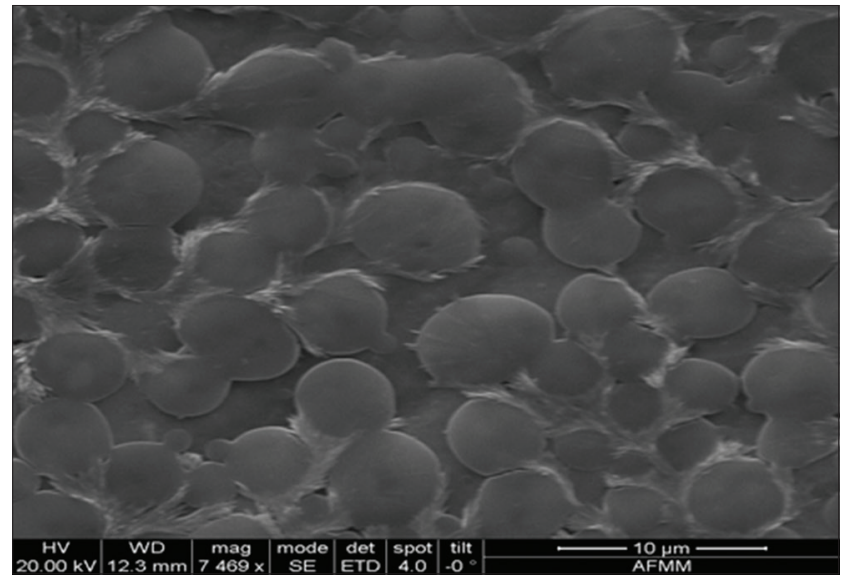

Fig. 13: Scanning electron microscopy images of almotriptan loaded gelatin nanoparticles
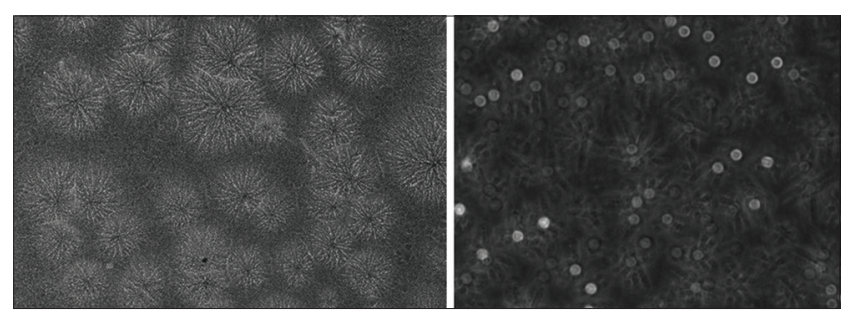

Fig. 14: Scanning electron microscopy images of albumin and almotriptan loaded albumin nanoparticles

The effect of different drug to polymer ratios and the amount of cross linker, on average PS, zeta potential, polydispersity index, and EE of NPs is shown in Tables 7-10. The PS ranged from $48 \mathrm{~nm}$ (Formulation $A_{A 5}$ ) to $176 \mathrm{~nm}$ (Formulation $A_{G 9}$ ) which was prepared using the higher amount of polymer in the organic phase. The increase in the amount of polymer in the organic phase resulted in significant differences in PS.

The shape and surface morphology of both the formulations were visualized by SEM. The NPs were spherical in shape and had a smooth surface (Figs. 13 and 14).

\section{CONCLUSION}

Triptan-loaded albumin and G-NPs was successfully formulated via the coacervation technique. Among all the formulations, formulation $A_{A 5}$ containing drug and albumin in the ratio of $1: 2$ and $200 \mu \mathrm{l}$ of $4 \%$ glutaraldehyde was selected as the optimum formulation. The optimum $\mathrm{A}_{\mathrm{A} 5}$ formulation had the PS of $48.8 \mathrm{~nm}$, polydispersity index of 0.51 and zeta potential of $+29.5 \mathrm{mV}$, with the high $\mathrm{EE} 67 \pm 1.1 \%$ in NP formulation.

\section{REFERENCES}

1. Kassem A, Labib GS. Flash dissolving sublingual almotriptan malate lyotabs for management of migraine. Int J Pharm Pharm Sci 2017;9:125-31.

2. Keam S, Goa K, Figgitt D. Almotriptan: A review of its use in a migraine. Headache. J Head Face Pain 2003;43:300-1.

3. Mark H, Kim P. Drug Class Review Triptans. Vol. 4. Portland, OR: Oregon Health and Science University; 2009. p. 5-6.

4. Yadav BV, Ravichandiran V, Kumar SS. Preparation and characterization of gemcitabine loaded MPEG-PCL polymeric nanoparticles for improved transportation across blood brain barrier. Int J Pharm Pharm Sci 2016;8:83-90.

5. Mistry A, Stolnik S, Illum L. Nanoparticles for direct nose-to-brain delivery of drugs. Int J Pharm 2009;379(1):146-57.

6. Wong HL, Wu XY, Bendayan R. Nanotechnological advances for the delivery of CNS therapeutics. Adv Drug Deliv Rev 2012;64(7):686-700.

7. Jawahar N. Development and characterization of PLGA nanoparticles containing carvedilol. J Pharm Sci Res 2009;1(3):123-8.

8. Saikat D, Rinti B, Jayesh B. Aspirin Loaded Albumin Nanoparticles by Coacervation: Implications in Drug Delivery. Vol. 18. Bombay: School of Biosciences and Bioengineering, Indian Institute of Technology; 2005. p. 202-11.

9. Ankarao A, Vasu VN, Hanumantha KR. Formulation and in-vitro evaluation of oral sustained release nano particulate delivery system of carvedilol. Int J Res Pharm Biomed Sci 2012;3:923-4.

10. Young W, Yong HK. Preparation and cytotoxicity comparison of Type A gelatin nanoparticles with recombinant human gelatin nanoparticles. Macromol Res 2009;17(7):464-8.

11. Azimi B, Nourpanah P, Rabiee M, Arbab S. Producing gelatin nanoparticles as delivery system for bovine serum albumin. Iran Biomed J 2014;18(1):34-40.

12. Azarmi S, Huang $\mathrm{Y}$, Chen $\mathrm{H}$, McQuarrie S, Abrams D, Roa W, et al. Optimization of a two-step desolvation method for preparing gelatin nanoparticles and cell uptake studies in 143B osteosarcoma cancer cells. J Pharm Pharm Sci 2006;9(1):124-32.

13. Calvo P, Remunan-Lopez C, Vila-Jato JL, Alonso MJ. Novel hydrophilic chitosan polyethylene oxide nanoparticles as protein carriers. J Appl Polym Sci 1997;63(1):125-32

14. Gaurav K. Mannosylated gelatin nanoparticles bearing isoniazid for selective management of tuberculosis. J Drug Target 2011;19(3):219-27. 